\title{
Diabetes and risk of community-acquired Staphylococcus aureus bacteremia: a population-based case-control study
}

\author{
Jesper Smit 1,2,3, Mette Søgaard ${ }^{3}$, Henrik Carl Schønheyder ${ }^{1,4}$, Henrik Nielsen ${ }^{2,4}$, \\ Trine Frøslev ${ }^{3}$ and Reimar Wernich Thomsen ${ }^{3}$ \\ ${ }^{1}$ Department of Clinical Microbiology, Aalborg University Hospital, Aalborg, Denmark, ${ }^{2}$ Department of \\ Infectious Diseases, Aalborg University Hospital, Aalborg, Denmark, ${ }^{3}$ Department of Clinical \\ Epidemiology, Aarhus University Hospital, Aalborg, Denmark and ${ }^{4}$ Department of Clinical Medicine, \\ Aalborg University, Aalborg, Denmark
}

\author{
Correspondence \\ should be addressed \\ to J Smit \\ Email \\ jesm@rn.dk
}

\begin{abstract}
Objective: Patients with diabetes may experience higher risk of Staphylococcus aureus bacteremia (SAB) than patients without diabetes due to decreased immunity or coexisting morbidities. We investigated the risk of communityacquired (CA) SAB in persons with and without diabetes.

Design: Using population-based medical databases, we conducted a case-control study of all adults with first-time CA-SAB and matched population controls in Northern Denmark, 2000-2011.

Methods: Based on conditional logistic regression, we computed odds ratios (ORs) of CA-SAB according to diabetes. We further assessed whether the risk of CA-SAB differed according to various diabetes-related characteristics (e.g. duration of diabetes, glycemic control, and presence of diabetes complications).

Results: We identified 2638 patients with incident CA-SAB, of whom 713 (27.0\%) had diabetes, and 26379 matched population controls ( 2495 or $9.5 \%$ with diabetes). Individuals with diabetes had a substantially increased risk of CA-SAB compared with population controls (adjusted $\mathrm{OR}=2.8$ (95\% confidence interval $(\mathrm{Cl})$ : $2.5-3.1)$ ). Duration of diabetes of $\geq 10$ years and poor glycemic control conferred higher risk estimates, with an adjusted $\mathrm{OR}=2.3$ (95\% Cl: 1.9-2.7) for diabetes with $\mathrm{Hba} 1 \mathrm{c}<7 \%$ ( $<53 \mathrm{mmol} / \mathrm{mol}$ ) and an adjusted $\mathrm{OR}=5.7$ (95\% Cl: 4.2-7.7) for diabetes with $\mathrm{Hba} 1 \mathrm{c} \geq 9 \%$ ( $\geq 75 \mathrm{mmol} / \mathrm{mol})$. The risk of CA-SAB was particularly high in patient with diabetes complications: adjusted $\mathrm{OR}=5.5(95 \% \mathrm{Cl}: 4.2-7.2)$ with presence of microvascular complications and $\mathrm{OR}=7.0$ (95\% Cl: 5.4-9.0) with combined macro- and microvascular complications.

Conclusions: Diabetes is associated with a substantially increased risk of CA-SAB, particularly in patients with diabetes of long duration, poor glycemic control, and diabetes complications.

\section{Introduction}

Staphylococcus aureus remains a leading cause of bacteremia associated with a 30 -day mortality of $20-40 \%(1,2)$. Diabetes is an increasingly common disease with detrimental effects on almost all organ systems and patients' quality of life $(3,4)$. Patients with diabetes may have increased susceptibility to $S$. aureus bacteremia $(\mathrm{SAB})$ for a number of reasons including tissue hyperglycemia and decreased oxygenation, and generally reduced immunity $(5,6)$. High age, coexisting morbidities and diabetes complications may further increase the risk of SAB (6).
() 2016 European Society of Endocrinology Printed in Great Britain
In previous studies of $\mathrm{SAB}$, diabetes has been noted as an underlying disease in $20-32 \%$ of patients $(7,8,9,10)$. Nevertheless, few clinical data exist concerning the association between diabetes and SAB, and to our knowledge, no prior study has investigated diabetes as a risk factor for $S A B$ as the main exposure. In previous studies, patients with diabetes have been identified using nonvalidated methods, and diabetes has been treated as one entity disregarding the duration of disease, quality of glycemic control, and presence of diabetes complications

Published by Bioscientifica Ltd 
$(11,12,13,14)$. In addition, the majority of former studies has been based on small and selected study populations $(n<250)(11,13,14)$.

Detailed information on the association between diabetes and $\mathrm{SAB}$ may extend our understanding of risk factors for $\mathrm{SAB}$ and help to optimize preventive measures for the growing group of persons with diabetes. Therefore, we conducted a population-based case-control study to investigate whether diabetes is associated with an increased risk of community-acquired $\mathrm{SAB}$ (CA-SAB). In addition, we ascertained the risk of CA-SAB according to various characteristics of patients with diabetes (e.g. diabetes type, duration of diabetes, and presence of diabetes complications), and we explored whether the risk of CA-SAB differed according to sex, age, and level of comorbidity.

\section{Methods}

\section{Setting}

This case-control study was conducted in Northern Denmark (catchment population $\sim 1.8$ million inhabitants) between January 12000 and December 312011 using population-based medical databases with routinely recorded data. Denmark has a tax-supported healthcare system providing free and unrestricted access to medical care for the entire Danish population. All Danish citizens are assigned a unique identification number (the civil registration number) upon birth or immigration, which allows unambiguous electronic linkage across the data sources $(15,16)$.

\section{Patients with $S$. aureus bacteremia}

Patients hospitalized with CA-SAB were identified in the databases of four regional departments of clinical microbiology from 1995 onwards (information on blood culture practice and susceptibility testing is provided in the Supplementary Material 1, see section on supplementary data given at the end of this article). We restricted inclusion to patients $\geq 15$ years with presence of $\geq 1$ positive blood cultures with $S$. aureus as the sole isolate. Compared to the general population, patients with previous $\mathrm{SAB}$ are at increased risk of reinfection with SAB (17). Therefore, we only included patients with incident $S A B$, defined as no prior SAB diagnosis within at least 5 years of the current admission.

CA-SAB was defined as SAB in patients, in whom one or more positive blood cultures had been obtained within the first 2 days of admission. If the positive blood culture had been obtained $>2$ days after admission, the infection was considered to be hospital-acquired, and the patient was excluded. The subset of CA-SAB patients with recent contacts to healthcare before the current admission was further classified as healthcare-associated SAB (HCA-SAB) if one or more of the following criteria were fulfilled: $\geq 1$ hospital admission, $\geq 1$ contacts to hospital outpatient surgical clinics, $\geq 1$ contacts to clinics of hematology, oncology or nephrology, all within a 30-day window of the current admission.

Information on recent healthcare contacts was provided by the Danish National Patient Registry (DNPR) (18). The DNPR has tracked all admissions to Danish hospitals since 1977 and all visits to hospital outpatient clinics since 1995. Each record includes the dates of hospital admission and discharge, up to 20 discharge diagnoses, and information on surgical procedures.

\section{Selection of population controls}

The Danish Civil Registration System (DCRS), which is updated daily, keeps records on sex, age, residence, marital status, and vital status for all Danish residents $(15,16)$. We used this registry to randomly select 10 population controls for each $\mathrm{SAB}$ case on the date the first positive blood culture was drawn, matching by age, sex, and residence. Each control was assigned an index date identical to the SAB admission date for the matched case. We utilized the risk set sampling technique (19), i.e. eligible population controls had to be alive and at risk of a first hospitalization with $\mathrm{SAB}$ on the date the corresponding case was admitted.

\section{Patients with diabetes}

For both cases and controls, we identified patients with diabetes using a previously validated algorithm (20) that incorporates three databases: the DNPR, the LABKA Database (21), and The Aarhus University Prescription Database (AUPD) (22). Patients with a discharge or outpatient diagnosis of diabetes registered at any time before the index date were identified in the DNPR. The LABKA database (CSC Scandihealth, Denmark) holds laboratory test results from patients in Northern Denmark for the entire study period, including the exact time of blood sample collection (21). Using this database, we identified patients with a glycosylated hemoglobin A1c (HbA1c) level of $6.5 \%$ (48 $\mathrm{mmol} / \mathrm{mol})$ or more measured at any time predating the index date. The AUPD contains 
information on all filled prescriptions in the study area in accordance with the anatomical therapeutic classification (ATC) (22). This database permitted identification of patients with at least one recorded prescription for any antidiabetic drug at any time before the index date (diagnostic, laboratory, and ATC codes are provided in the Supplementary Material 2). Patients with diabetes diagnosed before age 30 years, using insulin monotherapy, and with no history of oral anti diabetes medication were classified as type 1 diabetes. The remaining patients with diabetes were classified as type 2 diabetes.

\section{Characteristics of patients with diabetes}

We computed the duration of diabetes as the time elapsed between the first record of diabetes in any of the three registers and the date the first positive blood culture was sampled. To assess the level of preadmission glycemic control, we obtained data on all Hba1c measurements within 12 months of the index date. One or more Hba1c measurements were available for $515(72 \%)$ of the 713 cases with CA-SAB, and 1819 (73\%) of the 2495 controls with diabetes. The most recent Hba1c measurement before the index date was used in our analyses. Based on in- or outpatient contacts registered in the DNPR, we obtained data on the presence of macrovascular-, and microvascular complications, including in the latter indication of diabetes-associated renal disease in previous laboratory tests (defined by two separate dates with urinary albumin tests above the cut-off for microalbuminuria). Using the DNPR, we also identified patients with diabetes with conditions related to diabetic foot ulcers (i.e. neuropathy and/or peripheral atherosclerosis or vascular disease) and diabetes patients with previous lower-extremity ulcer diagnoses or ulcer-related procedures, as described previously (23). Preadmission renal function was ascertained using the most recent creatinine measurement from a general practitioner or an outpatient hospital clinic 1 year to 7 days before the index date (available for $78 \%$ of patients). We computed estimated glomerular filtration rates (eGFR) applying the four-variable version of the Modification of Diet in Renal Disease equation (24) (equation provided in the Supplementary Material 2).

\section{Demographics, comorbidity, and medication}

Using the DNPR (18), we retrieved all diagnoses recorded up to 10 years before the index date to identify previous morbidity included in the Charlson Comorbidity Index (CCI). The CCI is a validated scoring system for ascertaining patients' comorbid conditions in epidemiological studies and covers both the number and severity of 19 major disease categories $(25,26)$. Because diabetes constituted the exposure variable of interest, we removed this condition from the CCI and designated the index as a modified CCI $(\mathrm{m}-\mathrm{CCI})$. We classified patients as having a low $($ score $=0)$, an intermediate (score $=1-2$ ), or a high comorbidity level (score $>2$ ). Data on a number of comorbid conditions not included in the CCI, including hypertension, dialysis (within 30 days of the index date), osteoporosis, and conditions related to alcohol and drug abuse were also collated. Using the AUPD, we retrieved information on prescriptions filled before the index date: Any previous use of antihypertensive treatment, statins, anticoagulants, and use of immunosuppressive or antibiotic drugs within 30 days of the index date (laboratory, diagnostic, and ATC codes are provided in the Supplementary Material 2).

\section{Statistical analysis}

Characteristics of patients with and without diabetes were described in a contingency table. We used conditional logistic regression to compute crude and adjusted odds ratios (ORs) with corresponding 95\% confidence intervals (CIs) as a measure of relative risks of $\mathrm{SAB}$ among patients with and without diabetes. Diabetes exposure was further categorized by diabetes type, duration of diabetes $(<3, \geq 3-<6, \geq 6-<10$, $\geq 10$ years), level of glycemic control (Hba1c $<7 \%$ $(<53 \mathrm{mmol} / \mathrm{mol}), \geq 7-<8 \%(\geq 53-<64 \mathrm{mmol} / \mathrm{mol})$, $\geq 8-<9 \%(\geq 64-<75 \mathrm{mmol} / \mathrm{mol}), \geq 9 \%(\geq 75 \mathrm{mmol} /$ mol), unknown), diabetes complications (absent, microvascular, macrovascular, combined micro- and macrovascular, conditions related to diabetic foot ulcers, and previous lower-extremity ulcer diagnosis), and renal function before admission (eGFR $\left(\mathrm{mL} / \mathrm{min} / 1.73 \mathrm{~m}^{2}\right)>90$, $60-90,<60$, unknown). We adjusted for m-CCI score, alcohol-related conditions, marital status, any statin used predating the index date, and use of antibiotics within 30 days of the index date. To examine whether the risk of SAB differed among subsets of diabetes patients, we performed conventional logistic regression with additional adjustment for the matching factors and stratified the results by sex, age group (15-39, 40-59, $60-79,80+$ years), and m-CCI level $(0,1-2,>2)$. We conducted all analyses using Stata 11.2 for Windows (Statacorp). According to Danish legislation, individual informed consent is not required for registry-based studies. The project was approved by the Danish Data Protection Agency (ref. no. 2012-41-0942). 


\section{Results}

\section{Descriptive data}

During 2000-2011, we identified 2638 patients with incident CA-SAB and 26379 population controls. The subset of all CA-SAB patients with recent preadmission contacts to the healthcare system (HCA-SAB) constituted $42 \%$. MRSA was rarely observed (0.5\%). Characteristics of cases and controls are given in Table 1 . The median age of cases and controls was 69 years (interquartile range (IQR), 56-79), and $61 \%$ were males. CA-SAB patients were much more likely than population controls to have a history of hospital-diagnosed comorbidity, in particular congestive heart failure (13\% vs $4 \%)$, peripheral vascular disease $(12 \%$ vs $3 \%$ ), cancer ( $20 \%$ vs $7 \%$ ), and renal disease ( $17 \%$ vs $1 \%$ ). Cases were also more likely to have filled prescriptions for antibiotics, angiotensin-converting-enzyme inhibitors, beta blockers, and acetylsalicylic acid.

\section{Risk of S. aureus bacteremia}

A total of 713 (27.0\%) CA-SAB patients had diabetes, compared with 2495 (9.5\%) among population controls (Table 2). The unadjusted OR for incident CA-SAB in patients with diabetes compared with persons without diabetes was 3.7 (95\% CI: 3.4-4.1), and the adjusted OR was $2.8(2.5-3.1)$ (Table 2). The adjusted OR for CA-SAB was 7.2 (95\% CI: 3.9-13.0) in patients with type 1 diabetes compared with 2.7 (95\% CI: $2.4-3.0$ ) in patients with type 2 diabetes.

Compared with individuals without diabetes and persons with shorter duration of diabetes, patients with $\geq 10$ years of diabetes history experienced markedly increased the risk of CA-SAB (adjusted OR $=3.8$ (95\% CI: $3.2-4.6)$ ). The risk of CA-SAB rose gradually with successive increases in HbA1c levels. Compared with individuals without diabetes, the adjusted OR was 2.3 (95\% CI: 1.9-2.7) for patients with diabetes and an HbA1c level $<7 \%$ (<53 mmol/mol), 3.2 (95\% CI: $2.3-4.5)$ for patients with diabetes and HbA1c level $\geq 8$ to $<9 \%$ ( $\geq 64$ to $<75 \mathrm{mmol} / \mathrm{mol}$ ), and 5.7 (95\% CI: $4.2-7.7$ ) for patients with diabetes and an HbA1c level $\geq 9 \%$ ( $\geq 75 \mathrm{mmol} / \mathrm{mol}$ ). Diabetes complications influenced the risk of $\mathrm{CA}-\mathrm{SAB}$, as compared with persons without diabetes. Thus, the adjusted OR was 2.3 (95\% CI: 2.0-2.7) in patients with diabetes and no complications, 5.5 (95\% CI: $4.2-7.2)$ in patients with microvascular complications, 2.7 (95\% CI: 2.2-3.3) in patients with macrovascular complications, and 7.0 (95\% CI: 5.4-9.0) in persons with combined macro- and micro-vascular complications. Increased risk of CA-SAB was also evident among patients with diabetes and conditions related to diabetes foot ulcers (adjusted OR=4.9 (95\% CI: 3.7-6.6)), and patients with diabetes and a previous lower-extremity ulcer or ulcer-related procedure (adjusted $\mathrm{OR}=6.9$ (95\% CI: 5.4-8.8)). Decreased renal function also influenced the risk of CA-SAB with an adjusted OR of 4.2 (95\% CI: 3.5-5.1) in patients with diabetes and an eGFR $<60 \mathrm{~mL} / \mathrm{min} / 1.73 \mathrm{~m}^{2}$ compared with persons without diabetes.

Table 3 presents ORs according to diabetes stratified by age, sex, and comorbidity level. Female patients with diabetes appeared to experience a slightly increased risk of CA-SAB compared with males (adjusted ORs 3.2 (95\% CI: 2.6-3.8) and 2.5 (95\% CI: 2.2-2.9) respectively). The relative risk of CA-SAB decreased with advancing age and increasing level of comorbidity (Table 3 ).

\section{Discussion}

In this large population-based case-control study, diabetes was strongly associated with an increased risk of CA-SAB. Compared with patients without diabetes, the excess risk of CA-SAB was most pronounced among patients with type 1 diabetes, patients with $\geq 10$ years of diabetes history, patients with poor glycemic control, and patients with diabetes complications, in particular microvascular disease. In addition, the relative impact of diabetes was most pronounced in younger patients and in patients without coexisting morbidities.

Our results extend the limited existing knowledge on the risk of CA-SAB in patients with diabetes $(11,12$, $13,14)$. In an Italian case-control study of 165 patients with SAB, Bassetti et al. (11) observed an OR of 6.21 (1.62-23.77) from diabetes in multivariate analysis. In a Canadian surveillance cohort study (12) including 1508 SAB patients, the authors reported a substantial risk of SAB associated with diabetes (relative risk $(\mathrm{RR})=10.6$ (95\% CI: 9.3-11.9)). In two prior studies $(13,14)$, the investigators assessed diabetes as a risk factor for invasive $S$. aureus infections, defined as the isolation of $S$. aureus from blood, cerebrospinal fluid, pleural or synovial fluid, or aseptically obtained deep-tissue aspirates or surgicaltissue samples. In a Swedish cohort study of 168 patients, Jacobsson et al. (14) observed a RR of 8.2 (95\% CI: 6-12) for invasive $S$. aureus infections in patients with diabetes as compared with patients without. This was supported by results from a Canadian study cohort (13) $(n=264)$, where 
Table 1 Characteristics of cases with incident, community-acquired Staphylococcus aureus bacteremia and population controls, Northern Denmark, 2000-2011.

\begin{tabular}{l}
\hline \\
\hline Numbers (\%) \\
Diabetes \\
Absent \\
Present \\
Age, median (IQR) \\
$\geq 15-39$ years \\
$40-59$ years \\
$60-79$ years \\
$\geq 80$ years \\
Sex \\
Men \\
Women \\
Marital status \\
Married \\
Divorced or widowed \\
Never married \\
Selected comorbid conditions \\
Former myocardial infarction \\
Congestive heart failure \\
Peripheral vascular disease \\
Chronic pulmonary disease \\
Moderate to severe renal disease \\
Any solid cancer \\
Hypertension \\
Dialysis within 30 days of the index date \\
Conditions related to alcohol abuse \\
Conditions related to drug abuse \\
Modified Charlson Comorbidity Index score \\
Low (0) \\
Intermediate ( $1-2$ ) \\
High ( 2 ) \\
Medication use before the index date \\
Immunosuppressive therapya \\
Systemic antibiotic therapy \\
ACE inhibitors \\
Beta blockers \\
eGFR $>90$ mL/min/1.73 $\mathrm{m}^{2}$ \\
Statins ${ }^{\mathrm{b}}$ \\
eGFR $60-90$ mL/min/1.73 $\mathrm{m}^{2}$ \\
eGFR 60 mL/min/1.73 $\mathrm{m}^{2}$ \\
eGFR ${ }^{\mathrm{b}}$ missing \\
\hline
\end{tabular}

\begin{tabular}{l}
\hline Cases \\
\hline $2638(9.1 \%)$ \\
$1925(73.0)$ \\
$713(27.0)$ \\
$69.0(56.2-79.3)$ \\
$233(8.9)$ \\
$605(22.9)$ \\
$1182(44.8)$ \\
$618(23.4)$ \\
$1616(61.3)$ \\
$1022(38.7)$ \\
$1270(48.1)$ \\
$886(33.6)$ \\
$482(18.3)$ \\
$220(8.3)$ \\
$348(13.2)$ \\
$328(12.4)$ \\
$363(13.8)$ \\
$436(16.5)$ \\
$515(19.5)$ \\
$651(24.7)$ \\
$251(9.5)$ \\
$235(8.9)$ \\
$73(2.8)$ \\
$810(30.7)$ \\
$1012(38.4)$ \\
$816(30.9)$ \\
$28(1.1)$ \\
$536(20.3)$ \\
$1086(41.2)$ \\
$1035(39.2)$ \\
$1121(42.5)$ \\
$625(23.7)$ \\
$369(14.0)$ \\
$605(22.9)$ \\
$802(30.4)$ \\
$862(32.7)$ \\
\hline
\end{tabular}

\begin{tabular}{l}
\hline Controls \\
\hline $26379(90.9)$ \\
$23884(90.5)$ \\
$2495(9.5)$ \\
$69.0(56.3-79.3)$ \\
$2340(8.9)$ \\
$6009(22.8)$ \\
$11838(44.9)$ \\
$6192(23.5)$ \\
$16159(61.3)$ \\
$10220(38.7)$ \\
$15059(57.1)$ \\
$7742(29.4)$ \\
$3578(13.56)$ \\
$1037(3.93)$ \\
$960(3.6)$ \\
$889(3.4)$ \\
$1491(5.7)$ \\
$290(1.1)$ \\
$1778(6.7)$ \\
$3016(11.4)$ \\
$21(0.1)$ \\
$398(1.5)$ \\
$49(0.2)$ \\
$19035(72.2)$ \\
$6067(23.0)$ \\
$1277(4.8)$ \\
$54(0.2)$ \\
$1252(4.8)$ \\
$6768(25.7)$ \\
$6470(24.5)$ \\
$7771(29.5)$ \\
$4633(17.6)$ \\
$2115(8.0)$ \\
$6312(23.9)$ \\
$3328(12.6)$ \\
$14624(55.4)$ \\
\hline
\end{tabular}

Patients with type 1 diabetes $=5.9 \%$

${ }^{\text {aAny }}$ use within 30 days of the index date; ${ }^{b} A n y$ previous use before the index date; ${ }^{c}$ Measured within 1 year to 7 days before the index date. IQR, interquartile range; MRSA, methicillin resistant Staphylococcus aureus.

ACE inhibitors, angiotensin-converting-enzyme inhibitors; eGFR, estimated glomerular filtration rate; CRP, C-reactive protein.

the investigators observed a considerable risk of invasive $S$. aureus infections in patients with diabetes $(\mathrm{RR}=7.0$ (95\% CI: 5.0-9.7)).

Nevertheless, some limitations should be taken into account in the interpretation of these previous results: Small and selected study populations $(11,13,14)$, limited numbers of patients with diabetes $(11,13,14)$, incomparability of characteristics of cases and controls (11), or insufficient adjustment for concurrent comorbid conditions (12) could partly explain the observations. Furthermore, in contrast to our study, none of the previous studies assessed whether the risk of SAB differed according to characteristics of patients with diabetes or according to age, sex, and comorbidity level. 
Table 2 Crude and adjusted odds ratios (ORs) for community-acquired Staphylococcus aureus bacteremia associated with diabetes. Data are presented as $n(\%)$.

\begin{tabular}{l}
\hline Exposure \\
\hline Diabetes \\
Absent \\
Present \\
Diabetes type \\
Diabetes absent \\
Type 1 \\
Type 2 \\
Duration of diabetes \\
Diabetes absent \\
$<3$ years \\
$\geq 3$ to $<6$ years \\
$\geq 6$ to 10 years \\
$\geq 10$ years \\
HbA1c \\
Diabetes absent \\
$<7 \%(<53$ mmol/mol) \\
$\geq 7$ to $<8 \%$ ( $\geq 53$ to $<64$ mmol $/ \mathrm{mol})$ \\
$\geq 8$ to $<9 \%$ ( $\geq 64$ to $<75$ mmol/mol) \\
$\geq 9 \%$ ( $\geq 75$ mmol/mol) \\
HbA1c unknown \\
Diabetes complications \\
Diabetes absent \\
No complications \\
Microvascular only \\
Macrovascular only \\
Macro- and micro-vascular \\
Conditions related to diabetic foot \\
ulcers ${ }^{b}$ \\
Previous lower-extremity ulcerc \\
Renal function before the index date \\
Diabetes absent \\
eGFR $>90$ \\
eGFR $60-90$ \\
eGFR $<60$ \\
eGFR missing \\
\end{tabular}

\begin{tabular}{c}
\hline Cases \\
\hline $1925(73.0)$ \\
$713(27.0)$ \\
$1925(73.0)$ \\
$40(1.5)$ \\
$673(25.5)$ \\
$1925(73.0)$ \\
$176(6.7)$ \\
$144(5.5)$ \\
$123(4.7)$ \\
$270(10.2)$ \\
\\
$1925(73.0)$ \\
$245(9.3)$ \\
$100(3.8)$ \\
$69(2.6)$ \\
$101(3.8)$ \\
$198(7.5)$ \\
$1925(73.0)$ \\
$248(9.4)$ \\
$105(4.0)$ \\
$205(7.8)$ \\
$155(5.9)$ \\
$154(5.8)$ \\
$244(9.3)$ \\
$1925(73.0)$ \\
$90(3.4)$ \\
$155(5.9)$ \\
$311(11.8)$ \\
$157(6.0)$ \\
\end{tabular}

\begin{tabular}{c}
\hline Population controls \\
\hline $23884(90.5)$ \\
$2495(9.5)$ \\
$23884(90.5)$ \\
$29(0.1)$ \\
$2466(9.4)$ \\
23884 \\
$766(2.9)$ \\
$596(2.3)$ \\
$532(2.0)$ \\
$601(2.3)$ \\
$23884(90.5)$ \\
$1029(3.9)$ \\
$454(1.7)$ \\
$169(0.6)$ \\
$167(0.6)$ \\
$676(2.6)$ \\
$23884(90.5)$ \\
$1301(4.9)$ \\
$268(1.0)$ \\
$722(2.7)$ \\
$204(0.8)$ \\
$188(0.7)$ \\
$242(0.9)$ \\
$23884(90.5)$ \\
$364(1.4)$ \\
$906(3.4)$ \\
$686(2.6)$ \\
$539(2.0)$ \\
\end{tabular}

\begin{tabular}{|c|c|}
\hline Unadjusted OR $(95 \% \mathrm{Cl})$ & Adjusted $^{\text {a }}$ OR $(95 \% \mathrm{Cl})$ \\
\hline 1.0 (ref.) & 1.0 (ref.) \\
\hline $3.7(3.4-4.1)$ & $2.8(2.5-3.1)$ \\
\hline 1.0 (ref.) & 1.0 (ref.) \\
\hline $16.5(10.0-27.1)$ & $7.2(3.9-13.0)$ \\
\hline $3.6(3.2-3.9)$ & $2.7(2.4-3.0)$ \\
\hline 1.0 (ref.) & 1.0 (ref.) \\
\hline $3.0(2.5-3.6)$ & $2.5(2.0-3.0)$ \\
\hline $3.1(2.6-3.8)$ & $2.6(2.1-3.2)$ \\
\hline $3.1(2.5-3.8)$ & $2.1(1.7-2.7)$ \\
\hline $5.9(5.0-6.9)$ & $3.8(3.2-4.6)$ \\
\hline 1.0 (ref.) & 1.0 (ref.) \\
\hline $3.1(2.7-3.7)$ & $2.3(1.9-2.7)$ \\
\hline $2.9(2.3-3.6)$ & $2.2(1.7-2.9)$ \\
\hline $5.2(3.9-6.9)$ & $3.2(2.3-4.5)$ \\
\hline $7.8(6.0-10.0)$ & $5.7(4.2-7.7)$ \\
\hline $3.8(3.2-4.5)$ & $3.0(2.4-3.6)$ \\
\hline 1.0 (ref.) & 1.0 (ref.) \\
\hline $2.5(2.1-2.9)$ & $2.3(2.0-2.7)$ \\
\hline $5.0(4.0-6.4)$ & $5.5(4.2-7.2)$ \\
\hline $3.9(3.3-4.6)$ & $2.7(2.2-3.3)$ \\
\hline $10.1(8.1-12.6)$ & $7.0(5.4-9.0)$ \\
\hline $11.1(8.9-13.9)$ & $4.9(3.7-6.6)$ \\
\hline $13.2(10.9-15.9)$ & $6.9(5.4-8.8)$ \\
\hline 1.0 (ref.) & 1.0 (ref.) \\
\hline $3.2(2.5-4.0)$ & $2.2(1.7-3.0)$ \\
\hline $2.3(1.9-2.7)$ & $1.8(1.4-2.2)$ \\
\hline $6.1(5.3-7.1)$ & $4.2(3.5-5.1)$ \\
\hline $3.7(3.1-4.5)$ & $3.8(3.0-4.7)$ \\
\hline
\end{tabular}

aAdjusted for: conditions included in the modified Charlson Comorbidity Index (excluding the morbidity in question), marital status, alcohol-related conditions, any statin use predating the index date, and antibiotic therapy within 30 days of the index date; batients with diabetes and a previous diagnosis of neuropathy and/or peripheral vascular disease; ${ }^{~}$ Patients with diabetes and a previous lower-extremity ulcer diagnosis or ulcer-related procedure.

$\mathrm{Cl}$, confidence interval; HbA1c, Hemoglobin A1c; eGFR, estimated glomerular filtration rate $\left(\mathrm{mL} / \mathrm{min} / 1.73 \mathrm{~m}^{2}\right)$.

A number of different pathophysiological mechanisms may explain the observed increased risk of CA-SAB associated with diabetes. Diabetes and SAB share several risk factors including high age and comorbidity (6). Adjusting for comorbid conditions attenuated the association between diabetes and CA-SAB, suggesting that part of the risk associated with diabetes is conveyed by the burden of multiple morbidities and concomitant general frailty.

In our study, the excess risk of SAB was particularly evident in patients with diabetes complications. Decreased skin barriers may allow staphylococci access to adjoining tissues (27) or, at the most severe end of the spectrum, the blood stream $(1,2)$. Our findings of high ORs associated with diabetic foot ulcers may support this mechanism. Moreover, renal disease is a well-known risk factor for SAB (28), which may be of particularly importance in patients with concomitant diabetes, as indicated by the OR of 4.2 (95\% CI: $3.5-5.1)$ observed in patients with an eGFR $<60 \mathrm{~mL} / \mathrm{min} / 1.73 \mathrm{~m}^{2}$.

Still, patients with diabetes may most likely experience increased susceptibility to CA-SAB for reasons other than concomitant morbidities including diabetes complications. Neutrophilic leukocytes constitute the primary cellular defense against $S$. aureus infections, yet chemotaxis, adhesion, and intracellular killing are impaired 
Table 3 Crude and adjusted odds ratios (ORs) for community-acquired Staphylococcus aureus bacteremia associated with diabetes according to sex, age, and level of comorbidity.

\begin{tabular}{|c|c|c|}
\hline & $\begin{array}{c}\text { Unadjusted OR } \\
(95 \% \mathrm{Cl})\end{array}$ & $\begin{array}{c}\text { Adjusted }^{\mathbf{a}} \text { OR } \\
(95 \% \mathrm{Cl})\end{array}$ \\
\hline Diabetes absent & 1.0 (ref.) & 1.0 (ref.) \\
\hline \multicolumn{3}{|l|}{ Sex } \\
\hline Male & $3.7(3.2-4.1)$ & $2.5(2.2-2.9)$ \\
\hline Female & $3.9(3.3-4.6)$ & $3.2(2.6-3.8)$ \\
\hline \multicolumn{3}{|l|}{ Age } \\
\hline $15-39$ years & $11.9(6.6-21.3)$ & $4.0(1.6-9.7)$ \\
\hline $40-59$ years & $6.2(4.9-7.7)$ & $5.7(4.1-8.0)$ \\
\hline $60-79$ years & $3.6(3.1-4.1)$ & $2.6(2.2-3.1)$ \\
\hline $80+$ years & $2.7(2.2-3.2)$ & $2.3(1.8-2.8)$ \\
\hline \multicolumn{3}{|c|}{ Modified Charlson Comorbidity Index score } \\
\hline Low $(0)$ & $3.2(2.7-4.0)$ & $3.5(2.8-4.3)$ \\
\hline Intermediate $(1-2)$ & $2.2(1.9-2.5)$ & $2.5(2.1-3.0)$ \\
\hline High $(>2)$ & $2.0(1.6-2.4)$ & $2.2(1.7-2.7)$ \\
\hline
\end{tabular}

${ }^{a}$ Adjusted for: conditions included in the modified Charlson Comorbidity Index (except when stratified by this variable), age and gender (when stratified by comorbidity level), alcohol-related conditions, marital status, any statin use predating the index date, and antibiotic therapy within 30 days of the index date.

in patients with diabetes $(5,6,29)$. Furthermore, the impaired immunological response in patients with diabetes has been shown to be affected by the level of glycemic control $(30,31)$, which is in accordance with our observations of gradual increases in CA-SAB risk with successive increases in Hba1c levels.

The main strengths of our study include its considerable size, well-defined study population, and the use of routinely recorded clinical data. We excluded patients with hospital-acquired $\mathrm{SAB}$, which reduces confounding from major invasive procedures and concurrent diseases. In contrast to previous studies $(11,12,13,14)$, patients with diabetes were identified using a validated algorithm (20). Furthermore, we refined our analyses of diabetes as a risk factor for $\mathrm{SAB}$ by incorporating detailed information on various characteristics of patients with diabetes.

Limitations include the possibility that physicians may be more likely to admit patients with diabetes on suspicion of infection compared with patients without diabetes. Such surveillance bias would prompt an overestimation of the relative risk associated with SAB. However, previous studies from our setting $(32,33)$ assessing the risk of pneumococcal bacteremia and pneumonia, respectively, demonstrated that preadmission antibiotic treatment, microbiological results, levels of inflammatory markers on admission, and proportion of patients with at least one blood culture were comparable among patients with and without diabetes. This suggests that there was no substantial bias associated with the management of patients with diabetes. Although our ascertainment of diabetes was based on three separate population-based registries, some persons with diabetes may have been missed, which would bias our results toward unity. Diabetic foot ulcers were not coded consistently with unique diagnostic codes during the study period. Thus, we used conditions related to foot ulcers and previous lower-extremity ulcer diagnoses, which might represent somewhat crude proxies. Nevertheless, both variables indicated a substantially increased risk of CA-SAB associated with diabetic foot ulcers, and we find it unlikely that misclassification alone could explain risk estimates of this magnitude. Finally, we lacked data to adjust for smoking and body mass index, which constitute a considerable limitation of the study. Still, these factors may be partly accounted for by the adjustment for lifestyle-related comorbidities in our analyses.

The low prevalence of MRSA in the study area ensured a clean focus on MSSA, but our results may not be directly applicable to settings with higher MRSA prevalence. Still, the results from our study may most likely be generalizable to other healthcare systems with equal access to healthcare and prescription medication including anti diabetes therapy.

In conclusion, persons with diabetes experienced an almost threefold increased risk of CA-SAB compared with persons without diabetes. Long diabetes duration, suboptimal glycemic control, and diabetes complications including renal disease further increased the risk of CA-SAB. These results emphasize the importance of improved preventive care for patients with diabetes, including optimized glycemic control, and particularly good infection surveillance among patients with long duration of diabetes and complications.

\section{Supplementary data}

This is linked to the online version of the paper at http://dx.doi.org/10.1530/ EJE-15-0023.

\section{Declaration of interest}

The authors declare that there is no conflict of interest that could be perceived as prejudicing the impartiality of the research reported.

\section{Funding}

This study was supported by research grants from The Heinrich Kopp, Hertha Christensen, and North Denmark Health Sciences Research Foundations. The Department of Clinical Epidemiology is a member of the Danish Centre for Strategic Research in Type 2 Diabetes (DD2), supported 
by the Danish Agency for Science (grant nos. 09-067009 and 09-075724). DD2 is also supported by the Danish Health and Medicines Authority, the Danish Diabetes Association, and an unrestricted donation from Novo Nordisk A/S. Partners in the DD2 project are listed on the project website at www.DD2.nu. The sponsors did not have a role in any phase of the study conduct.

\section{Author contribution statement}

J $\mathrm{S}$ designed the study and performed data management, analysis, and manuscript preparation. M S, H C S, and $\mathrm{H} \mathrm{N}$ contributed to the study concept and preparation, data interpretation, and manuscript review. T F provided statistical guidance. R W T contributed to study concept and design, critical analysis of the data, and manuscript review.

\section{References}

1 Kern WV. Management of Staphylococcus aureus bacteremia and endocarditis: progress and challenges. Current Opinion in Infectious Diseases 201023 346-358. (doi:10.1097/QCO.0b013e32833bcc8a)

2 Thwaites GE, Edgeworth JD, Gkrania-Klotsas E, Kirby A, Tilley R, Török ME, Walker S, Wertheim HFL, Wilson P, Llewelyn MJ et al. Clinical management of Staphylococcus aureus bacteraemia. Lancet Infectious Diseases 201111 208-222. (doi:10.1016/S14733099(10)70285-1)

3 Guariguata L, Whiting DR, Hambleton I, Beagley J, Linnenkamp U $\&$ Shaw JE. Global estimates of diabetes prevalence for 2013 and projections for 2035. Diabetes Research and Clinical Practice 2014103 161-175 (doi:10.1016/j.diabres.2013.11.002)

4 Whiting DR, Guariguata L, Weil C \& Shaw J. IDF diabetes atlas: global estimates of the prevalence of diabetes for 2011 and 2030. Diabetes Research and Clinical Practice 201194 311-321. (doi:10.1016/j. diabres.2011.10.029)

5 Koh GCKW, Peacock SJ, van der Poll T \& Wiersinga WJ. The impact of diabetes on the pathogenesis of sepsis. European Journal of Clinical Microbiology \& Infectious Diseases 201231 379-388.

6 Thomsen RW \& Mor A. Diabetes and risk of community-acquired respiratory tract infections, urinary tract infections, and bacteremia. Open Infectious Diseases Journal 20126 27-39. (doi:10.2174/18742793 01206010027)

7 Kaasch AJ, Barlow G, Edgeworth JD, Fowler VG, Hellmich M, Hopkins S, Kern WV, Llewelyn MJ, Rieg S, Rodriguez-Baño J et al. Staphylococcus aureus bloodstream infection: a pooled analysis of five prospective, observational studies. Journal of Infection 201468 242-251. (doi:10.1016/j.jinf.2013.10.015)

8 Lesens O, Methlin C, Hansmann Y, Remy V, Martinot M, Bergin C, Meyer P \& Christmann D. Role of comorbidity in mortality related to Staphylococcus aureus bacteremia: a prospective study using the Charlson Weighted Index of comorbidity. Infection Control \& Hospital Epidemiology 200324 890-896. (doi:10.1086/iche.2003.24. issue-12)

9 Forsblom E, Ruotsalainen E, Mölkänen T, Ollgren J, Lyytikäinen O \& Järvinen A. Predisposing factors, disease progression and outcome in 430 prospectively followed patients of healthcare- and communityassociated Staphylococcus aureus bacteraemia. Journal of Hospital Infection 201178 102-107. (doi:10.1016/j.jhin.2011.03.010)

10 Kaech C, Elzi L, Sendi P, Frei R, Laifer G, Bassetti S \& Fluckiger U. Course and outcome of Staphylococcus aureus bacteremia: a retrospective analysis of 308 episodes in a Swiss tertiary-care centre. Clinical Microbiology and Infection 200612 345-352. (doi:10.1111/ j.1469-0691.2005.01359.x)

11 Bassetti M, Trecarichi EM, Mesini A, Spanu T, Giacobbe DR, Rossi M, Shenone E, Pascale GD, Molinari MP, Cauda R et al. Risk factors and mortality of healthcare-associated and community-acquired
Staphylococcus aureus bacteraemia. Clinical Microbiology and Infection 201218 862-869. (doi:10.1111/j.1469-0691.2011.03679.x)

12 Laupland KB, Ross T \& Gregson DB. Staphylococcus aureus bloodstream infections: risk factors, outcomes, and the influence of methicillin resistance in Calgary, Canada, 2000-2066. Journal of Infectious Diseases 2008198 336-343. (doi:10.1186/s12879-014-0647-4)

13 Laupland KB, Church DL, Mucenski M, Sutherland LR \& Davies HD. Population-based study of the epidemiology of and the risk factors for invasive Staphylococcus aureus infections. Journal of Infectious Diseases 2003187 1452-1459. (doi:10.1086/jid.2003.187.issue-9)

14 Jacobsson G, Dashti S, Wahlberg T \& Andersson R. The epidemiology of and risk factors for invasive Staphylococcus aureus infections in western Sweden. Scandinavian Journal of Infectious Diseases 200739 6-13. (doi:10.1080/00365540600810026)

15 Pedersen CB. The Danish Civil Registration System. Scandinavian Journal of Public Health 201139 22-25. (doi:10.1177/1403494810387965)

16 Schmidt M, Pedersen L \& Sørensen HT. The Danish Civil Registration System as a tool in epidemiology. European Journal of Epidemiology 201429 541-549. (doi:10.1007/s10654-014-9930-3)

17 Wiese L, Mejer N, Schønheyder HC, Westh H, Jensen AG, Larsen AR, Skov R, Benfield T \& Danish Staphylococcal Bacteraemia Study Group. A nationwide study of comorbidity and risk of reinfection after Staphylococcus aureus bacteraemia. Journal of Infection $2013 \mathbf{6 7}$ 199-205. (doi:10.1016/j.jinf.2013.04.018)

18 Lynge E, Sandegaard JL \& Rebolj M. The Danish National Patient Register. Scandinavian Journal of Public Health 201139 30-33. (doi:10.1177/1403494811401482)

19 Wacholder S, McLaughlin JK, Silverman DT \& Mandel JS. Selection of controls in case-control studies. I. Principles. American Journal of Epidemiology 1992135 1019-1028. (doi:10.1007/BF00050867)

20 Thomsen RW, Hundborg HH, Lervang HH, Johnsen SP, Sørensen HT $\&$ Schønheyder HC. Diabetes and outcome of communityacquired pneumococcal bacteremia. Diabetes Care 200427 70-76. (doi:10.1097/01.CCM.0000207345.92928.E4)

21 Grann AF, Erichsen R, Nielsen AG, Frøslev T \& Thomsen RW. Existing data sources for clinical epidemiology: the clinical laboratory information system (LABKA) research database at Aarhus University, Denmark. Clinical Epidemiology 20111133.

22 Ehrenstein V, Antonsen S, Pedersen L. Existing data sources for clinical epidemiology: Aarhus University Prescription Database. Clinical Epidemiology 20102 273-279. (doi:10.2147/CLEP)

23 Sohn MW, Budiman-Mak E, Stuck RM, Siddiqui F \& Lee TA. Diagnostic accuracy of existing methods for identifying diabetic foot ulcers from inpatient and outpatient datasets. Journal of Foot and Ankle Research 20103 27. (doi:10.1186/1757-1146-3-27)

24 Levey AS, Coresh J, Greene T, Stevens LA, Zhang YL, Hendriksen S, Kusek JW, Van Lente F \& Chronic Kidney Disease Epidemiology Collaboration. Using standardized serum creatinine values in the modification of diet in renal disease study equation for estimating glomerular filtration rate. Annals of Internal Medicine 2006145 247-254. (doi:10.7326/0003-4819-145-4200608150-00004)

25 Charlson M, Pompei P \& Ales KL. A new method of classifying prognostic comorbidity in longitudinal studies: development and validation. Journal of Chronic Diseases 198740 373-383. (doi:10.1016/0895-4356(91)90008-W)

26 Thygesen SK, Christiansen CF, Christensen S, Lash TL \& Sørensen HT. The predictive value of ICD-10 diagnostic coding used to assess Charlson comorbidity index conditions in the population-based Danish National Registry of Patients. BMC Medical Research Methodology 201111 83. (doi:10.1186/1471-228811-83)

27 Otto M. How Staphylococcus aureus breaches our skin to cause infection. Journal of Infectious Diseases 2012205 1483-1485. (doi:10.1093/infdis/jis248) 
28 Nielsen LH, Jensen-Fangel S, Benfield T, Skov R, Jespersen B, Larsen AR, Østergaard L, Støvring H, Schønheyder HC \& Søgaard OS. Risk and prognosis of Staphylococcus aureus bacteremia among individuals with and without end-stage renal disease: a Danish population-based cohort study. BMC Infectious Diseases 2015156. (doi:10.1186/s12879-014-0740-8)

29 Schuetz P, Castro P \& Shapiro NI. Diabetes and sepsis: preclinical findings and clinical relevance. Diabetes Care 201134 771-778. (doi:10.2337/dc10-1185)

30 Peleg AY, Weerarathna T, McCarthy JS \& Davis TM. Common infections in diabetes: pathogenesis, management and relationship to glycaemic control. Diabetes/Metabolism Research and Reviews 200723 3-13 (doi:10.1002/(ISSN)1520-7560)
31 Benfield T, Jensen JS \& Nordestgaard BG. Influence of diabetes and hyperglycaemia on infectious disease hospitalisation and outcome. Diabetologia 200750 549-554. (doi:10.1007/s00125006-0570-3)

32 Thomsen RW, Hundborg HH, Lervang HH, Johnsen SP, Schønheyder HC \& Sørensen HT. Risk of community-acquired pneumococcal bacteremia in patients with diabetes: a populationbased case-control study. Diabetes Care 200427 1143-1147. (doi:10.1097/01.CCM.0000207345.92928.E4)

33 Kornum JB, Thomsen RW, Riis A, Lervang HH, Schønheyder HC \& Sørensen HT. Diabetes, glycemic control, and risk of hospitalization with pneumonia: a population-based case-control study. Diabetes Care 20088 1541-1545. (doi:10.2337/dc08-0138)

Received 8 January 2016

Revised version received 19 February 2016

Accepted 26 February 2016 
Downloaded from Bioscientifica.com at 04/26/2023 10:00:29AM via free access 